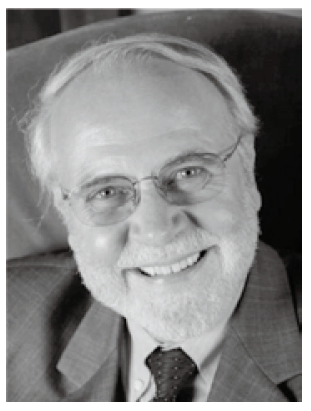

\title{
Science Challenges the System of Radiological Protection
}

\author{
Wolfgang WeIsS
}

During the last decade science has significantly contributed to our basic understanding of radiation risks. A vast amount of new evidence has become available which underpins and consolidates the assumptions of the system of radiological protection at low doses and low dose rates recommended by ICRP. On the other hand it has become evident that some scientific findings may challenge the system of radiological protection. There is considerable interest in the risks of hereditary effects and cancer and non-cancer diseases at specific workplaces and in the general population due to radiation exposure at low dose $(<200 \mathrm{mSv})$ and low dose rates like the ones observed in Japan in 2011.

Some of the fundamental questions for future scientific work are: Does the LNT model recommended by ICRP provide sufficient protection to all individuals and/or groups of the population? Is the application of a DDREF to extrapolate from acute to chronic exposures scientifically sound? Is there a need to distinguish between low dose and low dose-rate mechanisms when assessing stochastic effects? Are non-cancer risks contributing to the total detriment at low doses/low dose rates and, if so, how much? Can non-cancer risks be characterized as a stochastic phenomenon or can the underlying mechanism be assumed to be of deterministic nature?

In addition to these fundamental questions there are many practical issues to be answered such as: How can exposures resulting from diagnostic procedures in medicine be reduced without compromising the valuable information provided by imaging techniques like CT? How can radiation therapy treatment protocols be optimised in such a way as to minimise the risk of late effects in healthy tissue and of secondary cancers, given the increasing level of cancer survived?

According to the ongoing discussion in the scientific community, in policy-making organizations, and by stakeholders, future low-dose risk research should focus on specific health effects such as cancer - including secondary cancers, effects on the cardiovascular and the central nervous system, neurological effects, and lens opacity. The answers to some of the fundamental questions would require medium to long-term basic research; some of the practical questions could be answered on shorter time scales.

It is encouraging to observe that there are many research activities in this field world-wide. In order to utilize the existing scientific as well as the available financial resources in the most efficient way, strategic and transparent planning of research activities and of resource allocation is warranted. Planning should be based on an active dialogue between members the scientific community, policy makers, stakeholders, and end users of research projects. Different time scales (years to decades) should be considered in planning. As central elements efforts should be made to improve the co-ordination of research at the international level, to identify key research infrastructures and to contribute to their operation in a sustainable way. Major research programmes should also aim at establishing education and training programmes to overcome the degradation of competence in radiation research and radiation protection observed in many countries.

As central elements future research programmes should utilize new methodological approaches, promote the interaction with experts from outside the classical disciplines of radiobiology and epidemiology, and develop an agreed policy for post-publication sharing of data, biological samples and assessment tools. The key elements of a concept for post-publication sharing of data 
and tools have been described in the Rome Agenda (NATURE, Vol. 461, 10 September 2009). What's needed now is a policy to implement these ideas as requirements in future research programmes. Data bases and tissue banks have become resources of key importance in radiobiological and biomedical research. To better utilize these resources in the future, standards for the operation of scientific collections and corresponding performance indicators have to be defined and agreed and policies, guidelines and conditions have to be developed further that would ensure accessibility to such collections.

First steps in these directions are underway. In Europe, the MELODI association has been established in 2010. Supported by the EC funded research network DoReMi and by WHO programmes in the medical field, MELODI is committed to develop and implement in the years to come a strategic agenda for radiation research in the low dose/dose range as briefly described in this editorial. Contacts between professionals engaged in the MELODI work, the US Low Dose Programme, and similar activities in Japan have been established during recent years. It would be highly desirable if these informal contacts could be intensified and developed further.

\section{Wolfgang WeISS}

Prof. Dr. Wolfgang WeIss is head of the Department "Radiation Protection and Health" of the German Federal Office for Radiation Protection. He is acting as Chair of UNSCEAR (2011-2012), Vice President of MELODI, Vice Chair of Committee 4 of ICRP, and member of the bureau of OECD/NEA-CRPPH. 